\title{
16. SOME ASPECTS OF THE CREATION OF METEOR TRAINS AND RADIO-WAVE SCATTERING FROM THEM
}

\author{
K. V. KostyleV \\ (Radio Astronomy Department, Kazan University, U.S.S.R.)
}

\section{The Creation of Meteor Trains}

Small meteor particles are considered, which produce meteors with magnitudes between $+3^{\mathrm{m}}$ and $+10^{\mathrm{m}}$ to $+12^{\mathrm{m}}$, and for which the thermal conductivity may be assumed infinite. Fragmentation and air cap effects are neglected. The derivation is made taking into account radiation losses and temperature variation during the evaporation time. The temperature variation of the meteoroid is defined in this case by the equation:

$$
\frac{\mathrm{d} T}{\mathrm{~d} t}=\frac{S}{M C}\left[\frac{\rho V^{3}}{2}-\sigma\left(T^{4}-T_{0}^{4}\right) \beta_{1} \frac{S^{\prime}}{S}+\frac{Q}{S} \times \frac{\mathrm{d} M}{\mathrm{~d} t}\right] .
$$

Here $T$ is the temperature, $S$ is the cross-section, $M$ and $C$ the mass and thermal heat capacity, $V$ is the velocity, $S^{\prime}$ is the surface area, $T_{0}$ is the initial temperature, $\sigma$ is the Stefan-Boltzmann constant, $\beta_{1}$ is the coefficient of emissivity, $\rho$ is the air density, and $Q$ is the latent heat of evaporation of the meteor substance.

The decrement of mass is:

$$
\frac{\mathrm{d} M}{\mathrm{~d} t}=-S^{\prime} a T^{-1 / 2} e^{-b / T}-\lambda_{p} \frac{S}{2 Q} \rho V^{3} .
$$

The first term of Equation (2) describes the evaporation of the meteoroid and the second one - ablation (Levin, 1956; Kaščeev et al., 1967). It was assumed $\lambda_{p}=0.025$ (Kaščeev et al., 1967). The values $a$ and $b$ are characteristic of the substance.

In this article one-component particles are considered (or particles which have components of almost identical physical properties).

Equations (1) and (2) are solved together with the deceleration equation

$$
\frac{\mathrm{d} V}{\mathrm{~d} t}=-S \frac{\rho V^{2}}{M} .
$$

The set of Equations (1), (2), (3) was solved for velocities $12 \mathrm{~km} / \mathrm{sec}$ to $70 \mathrm{~km} / \mathrm{sec}$ and for values $M \delta^{2}$ ( $\delta=$ the particle density) from 4.5 to $2.8 \times 10^{5}$. The particle motion was considered from the heights where the particle temperatures could be assumed equal to that of an absolutely black sphere at a distance of one astronomical unit 
from the Sun. The particle movement was examined from this upper part of the path until its temperature reached the melting point. The modified set of Equations (1), (2), (3) was solved in which the Equation (2) was replaced by the expression describing the molten mass fraction over the melting part of the path. The variable values corresponding to $100 \%$ melting were the initial conditions for solving the main problem - meteor train creation.

The behaviour of a particle depends on the value of the expression

$$
A=V_{b 0}\left(M_{0} \delta^{2}\right)^{1 / 3}
$$

where $V_{b 0}$ is the vertical component of velocity.

If $A$ is rather small, the particle temperature reaches some maximum value $T_{\mathrm{m}}<T_{n}$ ( $T_{n}$ is the melting point) and then rapidly decreases due to the velocity decrease. The maximum temperature increases when $A$ increases. In case $T_{\mathrm{m}}>T_{n}$ the temperature only reaches the value $T_{n}$ and then does not vary till the end of the melting. If the particle energy is sufficiently large it will be fully melted. Then the temperature will increase. The evaporation, and energy expenditure for it, grow simultaneously. After an abrupt creation of the train the temperature increase slows down considerably. The particle mass rapidly decreases and at the train end the velocity decreases also. The heat energy acquired by the disappearing particle becomes less. The evaporation decreases rapidly as the train end is approached and the remainder of the particle decelerates and finally cools down and hardens. The value of the remaining mass depends greatly on the initial velocity.

Whether the meteor particle will go through all these stages or not depends on its initial mass, velocity, density, and other physical characteristics. Only those particles which form trains will be of interest. Those which have small energy and melt only partially, then decelerate and harden or do not reach the melting point, are micrometeorites and are treated in another paper.

The mathematical analysis of the set of Equations (1), (2), (3) and the results of the numerical solution have led us to the following conclusions:

(1) The evaporation process is rather stable and depends weakly both on the value, and on the possible variations, of the evaporation constants due to some 'negative feedback'.

(2) The meteor train length $L$ varies only slightly with the examined range of masses and velocities and is from $3.4 H \sec Z$ to $2.8 H \sec Z$ for velocities from $12 \mathrm{~km} / \mathrm{sec}$ to $20 \mathrm{~km} / \mathrm{sec}$ and from $2 \cdot 8 H \sec Z$ to $2 \cdot 4 H \sec Z$ for velocities greater than $20 \mathrm{~km} / \mathrm{sec}$. Here $H$ is the scale height.

(3) The shape of the evaporation curve is rather stable and for velocities greater than $20 \mathrm{~km} / \mathrm{sec}$ is well expressed by the equation (shape normalized relative to the maximum):

$$
f_{1}\left(\Delta t_{1}\right)=0.4472\left(e^{\Delta t_{1}}-e^{-\Delta t_{1}}\right)\left(1-e^{\Delta t_{1}-2 \cdot 75}\right)^{2} .
$$


Here $\Delta t_{1}=\left[\left(h-h_{1}\right) / H\right] \cos Z$, where $h_{1}$ is the melting end height, $Z$ is the zenith angle.

(4) The atmosphere pressure at the maximum of evaporation (if $\Delta t_{1}=1 \cdot 70$ ), taking into account $H$ from the COSPAR International Reference Atmosphere (1965), is expressed by the equation:

$$
P_{\mathrm{m}}=1.80 \times 10^{4}\left(1.66 \times 10^{-2} V_{0}+0 \cdot 87\right)\left(M_{0} \delta^{2}\right)^{0.28} V_{0}^{-2 \cdot 15}(\cos Z)^{0 \cdot 85} .
$$

$V_{0}$ and $M_{0}$ are the initial values of the velocity $(\mathrm{km} / \mathrm{sec})$ and mass (grams).

A comparison with the commonly used expression

$$
P_{\mathrm{m}_{1}}=1.30 \times 10^{4} \frac{\left(M_{0} \delta^{2}\right)^{1 / 3} \cos Z}{V_{0}^{2}}
$$

leads to the conclusion that the first equation gives heights appreciably smaller than Equation (7) for similar values. The height difference is about $13 \mathrm{~km}$ for velocities $70 \mathrm{~km} / \mathrm{sec}$ and $M \delta^{2}=2.8 \times 10^{-5}$. The reason is that the energy losses for radiation and particle heating during the evaporation were not taken into account in the derivation of (7). These effects are strong, especially for small particles.

(5) The atmosphere pressures at the train beginning, $P_{1}$, and end, $P_{2}$, are

$$
P_{1}=P_{\mathrm{m}} e^{-1 \cdot 70}, P_{2}=P_{\mathrm{m}} e^{1 \cdot 05} .
$$

(6) The deceleration is rather well expressed for a wide range of masses and velocities by formula:

where

$$
V=V_{0} f_{2}\left(\Delta t_{1}\right)
$$

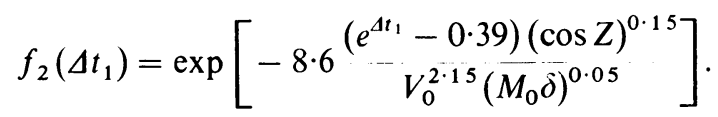

(7) If the ionization coefficient may be assumed

$$
\beta=\beta_{0} V^{n},
$$

the variation of the electron-line density $\alpha(\mathrm{el} / \mathrm{cm})$ along the meteor train is:

$$
\alpha=0.6 \frac{\beta_{0} V_{0}^{n}}{\mu H} M_{0} \cos Z \times f_{3}\left(\Delta t_{1}\right) .
$$

Here $f_{3}\left(\Delta t_{1}\right)=f_{1}\left(\Delta t_{1}\right)\left[f_{2}\left(\Delta t_{1}\right)\right]^{n}$ and $\mu$ is the average molecular weight of the particle substance.

\section{Radio-Wave Scattering from Underdense Meteor Trains}

The general problem of forward scattering by underdense trains is discussed. The variation of the ionization along the train is taken into account:

$$
\alpha=\alpha_{\mathrm{m}}^{\prime} f(S)
$$


where

$$
f(S)=e^{-l K S}\left(1-e^{-m K S}\right)^{n} .
$$

Here $K S=\left(h-h_{2}\right)(\cos Z) / H, h_{2}$ is the height of the train end, $l, m, n$ are positive values. Their choice allows representation of different kinds of ionization curve. Equation (5) is a combination of particular cases (10). If deceleration is taken into account, the problem becomes more complicated but its influence on the timeamplitude characteristics of the radio echo is weak. A finite time of train formation, the variation of the initial radius and diffusion coefficient, the presence of the train ends and variable position of the reflecting point are taken into account. Possible resonance phenomena, train distortion due to atmospheric turbulence, and anisotropic diffusion are not discussed. The aerial gain is assumed to be constant over the train. The radio echo power and phase are derived under these assumptions.

The finite expressions are:

The power in the receiving point ' $R$ ':

The echo phase

$$
P_{R}=\frac{1}{4} \bar{P}_{R}\left[Q^{2}(S)-Q^{2}\left(S_{1}\right)\right]
$$

$$
\operatorname{tg} \psi_{s}=\frac{|Q(S)| \sin \left(\gamma_{s}+\theta_{s}\right)-\left|Q\left(S_{1}\right)\right| \sin \left(\gamma_{1}+\theta_{1}\right)}{|Q(S)| \cos \left(\gamma_{s}+\theta_{s}\right)+\left|Q\left(S_{1}\right)\right| \cos \left(\gamma_{1}+\theta_{1}\right)} .
$$

Here $\bar{P}_{R}$ is the received power (Kazancev, 1961); $S_{1}$ is the train length, $S$ is the particle distance from the train end.

$$
Q^{2}(S)=\cos \Delta_{s} e^{-2 k_{2} r_{s}^{2}}\left[\left(\sum_{0}^{n} A_{i} e^{-(i m+l) K S} \bar{U}_{i s}\right)^{2}+\left(\sum_{0}^{n} A_{i} e^{-(i m+l) K S} \bar{V}_{i s}\right)^{2}\right],
$$

$Q\left(S_{1}\right) \approx 0$ if it is assumed that the ionization at the beginning of the train appears gradually:

$$
\cot \Delta_{s}=\frac{K_{3}}{C_{s} ; \quad K_{2}}=\begin{gathered}
4 \pi^{2} \\
\lambda^{2} \sec ^{2} \phi
\end{gathered} .
$$

$r_{s}$ is the initial radius of the train at the point situated at a distance $S$ from its end ( $S$ is the coordinate of the particle producing the train), $A_{i}$ are the coefficients in Equation (10) when raised to the $n$th power, $\bar{U}_{i s}$ and $\bar{V}_{i s}$ are special functions (Fadeeva and Terentev, 1954) depending on the coordinates

$$
\begin{aligned}
& X_{i}=-\sqrt{\frac{1}{2} \cos \Delta_{s}}\left[\left(\cos \frac{\Delta_{s}}{2}-\sin \frac{\Delta_{s}}{2}\right) \frac{a_{i s} L}{2 \sqrt{ } \pi}+\left(\cos \frac{\Delta_{s}}{2}+\sin \frac{\Delta_{s}}{2}\right) \sqrt{ } \pi\left(n_{0}-n_{s}\right)\right], \\
& Y_{i}=\sqrt{\frac{1}{2}} \overline{\cos \Delta_{s}}\left[\left(\cos \frac{\Delta_{s}}{2}+\sin \frac{\Delta_{s}}{2}\right) \frac{a_{i s} L}{2 \sqrt{ } \pi}-\left(\cos \frac{\Delta_{s}}{2}-\sin \frac{\Delta_{s}}{2}\right) \sqrt{ } \pi\left(n_{0}-n_{s}\right)\right] \text {. }
\end{aligned}
$$

In its turn

$$
K_{3}=\frac{\pi}{L^{2}} ; \quad C_{s}=K_{1} K D_{\mathrm{s}}+\frac{(\bar{m} K)^{2}}{2} K_{2} r_{s}^{2} ; \quad a_{i s}=(i m+l) K+K_{1} D_{s}+\bar{m} K K_{2} r_{s}^{2} ;
$$


$L$ is the half of the main Fresnel zone (Kazancev, 1961); $K_{1}=16 \pi^{2} /\left(V \lambda^{2} \sec ^{2} \Phi\right)$; $D_{s}$ is the diffusion coefficient at the point $S ; \bar{m}$ is from the expression of the initial radius variation (Greenhow and Hall, 1960); subscript 1 corresponds to the beginning and 2 to the end, so:

$$
r_{1}^{2}=r_{2}^{2} e^{\bar{m} K S_{1}}
$$

$n_{0}$ is the coordinate of the reflecting point (in units of $L$ ) from the train end and $n_{s}$ is the coordinate of the particle (in the same units); $\lambda$ is the wavelength; $2 \Phi$ is the scattering angle. If

$$
L>{ }_{a_{n 0}}^{2 \pi} \times \begin{gathered}
1-\operatorname{tg} \Delta_{0}^{\Delta_{0}} \\
2+\operatorname{tg} \Delta_{2}
\end{gathered},
$$

diffraction effects are practically absent. Then:

$$
\gamma_{s}=\frac{\varphi_{s}}{2}+\pi\left(n_{s}-n_{0}\right)^{2}+\frac{2 \pi}{\lambda}\left(R_{T}+R_{R}\right)
$$

Here $\varphi_{s}=\pi / 2-\Delta_{s}$; the last term expresses the constant phase shift depending on the distance between the reflecting point and the transmitter and the receiver $\left(R_{T}\right.$ and $R_{R}$ ).

Besides,

$$
\operatorname{tg} \theta_{s}=\sum_{0}^{n} A_{i} e^{-(i m+l) K S} \bar{V}_{i s} .
$$

The expressions mentioned above have a considerably simpler form for a wavelength of 3 or $4 \mathrm{~m}$. In the extreme 'classical' case of the uniformly ionized train they are transformed into usual expressions (McKinley, 1961).

The solution of the above equations leads to an echo typical of VHF (Evans, 1965 ) for the wavelength of $1 \sim 2 \mathrm{~m}$ (it depends on the train height.and the angle $\Phi$ ). The signal character changes considerably for short trains with rapidly varying ionization. The results of computer calculations show that the maximum amplitude occurs when $n_{\mathrm{m}}=0.7 \sim 0.8$ for wavelengths greater than $4 \mathrm{~m}$ and is approximated rather well by the formula (Peregudov, 1960):

$$
V_{\mathbf{m}} \sim e^{-K_{2} r_{0}^{2}} \frac{1-e^{-\delta}}{\delta}
$$

(where $\delta=R_{1} L D_{0}$ ), for different heights in the meteor region. It is also clear that the appearance of diffraction phenomena is practically impossible in the case of decay 
trains: the condition expressed by (14) is achieved after such a long period of time that the reflected signal amplitude becomes too small for the equipment sensitivity.

\section{References}

COSPAR (1965) Int. Ref. Atmosph., North-Holland Publ. Co., Amsterdam.

Evans, J.V. (1965) J. geophys. Res., 70, 5395.

Fadeeva, V.N., Terentev, N.M. (1954) Tablicy značenij integrala verojatnostej ot kompleksnogo argumenta, Moskva.

Greenhow, J.S., Hall, J.E. (1960) Mon. Not. R. astr. Soc., 121, 183.

Kaščeev, B. L., Lebedinec, V.N. (1961) Rezultaty Issled. MGG - Ionosfera i Meteory, No. 7, 1. Kaščeev, B. L., Lebedinec, V. N., Lagutin, M.F. (1967) Rezultaty Issled. MGR-Issled. Meteorov, No. 2,1 .

Kazancev, A.N. (1961) Meteornaja radiosvjaz na UKV, Moskva.

Lebedinec, V.N. (1963) Astr. Zu., 40, 719.

Levin, B.Ju. (1956) Fizičeskaja teorija meteorov i meteornoe veščestvo v solnečnoj sisteme, Akademizdat, Moskva.

McKinley, D.W.R. (1961) Meteor Science and Engineering, McGraw-Hill Book Co., New York. Peregudov, F. I. (1960) Harakteristika meteora kak radio-lokacionnoj celi, Tomsk. 